\title{
Tierschützerische Beurteilung des isolierten Frühabsetzens (Segregated Early Weaning, SEW) beim Schwein - eine Übersicht
}

\author{
Herrn Professor Dr. Dr. H. H. Sambraus zum 65. Geburtstag gewidmet
}

\begin{abstract}
Summary
Title of the paper: Welfare assessment of segregated early weaning (SEW) in pigs - a review

The technology of segregated early weaning (SEW) has been established primarily in large pig farm enterprises across North America. SEW is characterised by weaning piglets at days 7 to 21 of age (mostly between days 12 to 16) and isolated housing in nurseries and growing / finishing units (multi-site production with all-in all-out pig flow). The goal of this technology is to break the infection chain by utilising acquired maternal (passive) immunity from the dam before piglets develop their own active immunity in response to pathogens. A high standard of feeding and hygiene management facilitates a superior health status resulting in improved growth rates.

The EU-Report on the welfare status of intensively housed pigs (SCIENTIFIC VETERINARY COMMITTEE, 1997) suggests that the technology of SEW should be allowed only if it is demonstrated that any welfare advantages to sows and piglets outweigh the disadvantages. Recent research on the behaviour of piglets showed that piglets weaned before days 21 of age develop higher levels of nosing and chewing each other which persist into the grow/finish period. Longer-term consequences of SEW for the reproductive performance of sows are not yet sufficiently evaluated, although reduced nursing periods result into reduced litter sizes and piglet weights. That does, however, not exclude that the total number of litters/sow/year increases, resulting in greater number of piglets produced.

Considering the EU legislation laying down a minimum weaning age of 21 days, it is recommended that SEW technology could be implemented by utilising the appropriate health management features (multi-site production, all-in all-out). The benefits of isolated housing on health status and performance of pigs seem to outweigh the additional advantages of weaning piglets at a very early age $(<21$ days of age).
\end{abstract}

Key Words: pig, early weaning, animal welfare, behaviour, health, reproductive performance, productivity

\section{Zusammenfassung}

Das Produktionsverfahren des Segregated Early Weaning (SEW) hat sich insbesondere in Grossbetrieben Nordamerikas etabliert. Kennzeichen sind das Fruhabsetzen der Ferkel im Alter von 7 bis 21 Tagen (meist zwischen dem 12, u. 16. Lebenstag) und eine isolierte Aufzucht und Mast der Schweine an getrennten Standorten (MultiSite-Produktion nach dem Rein-Raus-Prinzip). Ziel dieser Produktionsweise ist die Unterbrechung von Erregerketten unter Ausnutzung der maternalen Immunităt, bevor sich das Immunsystem der Ferkel aktiv mit Erregern auseinander setzen muss. Bei entsprechendem Hygiene- und Futterungsmanagement lassen sich somit uber einen verbesserten Gesundheitsstatus hohe Wachstumsraten realisieren.

Der EU-Report zur Situation des Tierschutzes bei intensiv gehaltenen Schweinen (SCIENTIFIC VETERINARY COMMITTEE, 1997) beinhaltet eine Empfehlung zur Beurteilung des SEW: Danach sollte das SEW-Verfahren nur erlaubt werden, wenn nachgewiesen werden kann, dass die Vorteile des SEW bezulglich des Wohlbefindens von Sauen und Ferkeln gegenuber den Nachteilen uberwiegen. Neuere Verhaltensuntersuchungen belegen, dass unter 21 Tagen abgesetzte Ferkel sich vermehrt gegenseitig Besaugen und auch in spateren Haltungsabschnitten zu diesen Verhaltensabweichungen neigen. Langerfristige Auswirkungen auf die Sauenfruchtbarkeit sind noch nicht hinreichend untersucht. Verkürzte Săugezeiten wirken sich jedoch negativ auf die Wurfgröße und Ferkel- 
gewichte aus, was nicht ausschließt, dass sich insgesamt die Anzahl der Wurfe /Sau/ Jahr erhöht und damit auch die Anzahl der erzeugten Ferkel.

Bedingt durch das in der EU festgeschriebene Mindestabsetzalter von 21 Tagen wird daher empfohlen, unter Beachtung dieser Anforderungen bei konsequenter Umsetzung der Gesundheitsmanagementmaßnahmen (MultiSite-Produktion, Rein-Raus-Verfahren), das SEW-Prinzip zu nutzen. Die dabei erzielten Produktivitäts- und Gesundheitsvorteile scheinen gegenuber den vermeintlichen zusătzlichen Vorteilen des Absetzens zu einem fruheren Zeitpunkt nicht wesentlich nachzustehen.

Schlüsselwörter: Schwein, Frühabsetzen, Tierschutz, Verhalten, Gesundheit, Fruchtbarkeit, Produktivităt

\section{Einführung}

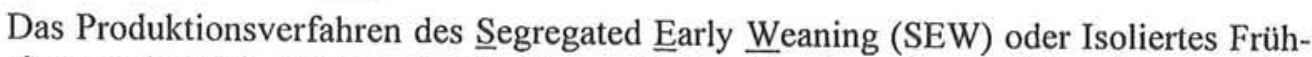
absetzen hat sich insbesondere in Grossbetrieben Nordamerikas etabliert. Kennzeichen sind das Frühabsetzen der Ferkel im Alter von 7 bis 21 Tagen (meist zwischen dem 12. u. 16. Lebenstag) und eine isolierte Aufzucht und Mast der Schweine an getrennten Standorten (Multi-Site-Produktion nach dem Rein-Raus-Prinzip). Ziel dieser Produktionsweise ist die Unterbrechung von Erregerketten unter Ausnutzung der maternalen Immunität, bevor sich das Immunsystem der Ferkel aktiv mit Erregern auseinander setzten muss. Das SEW hat sich historisch aus anderen Hygienemanagementverfahren entwickelt, die bereits in den 60'er Jahren praktiziert wurden (SPF-Prinzip = Specific $\underline{P}$ athogen Free). Auf der Grundlage der Untersuchungen von ALEXANDER et al. (1980) wurde als Vorstufe zum SEW in Zusammenarbeit mit dem Zuchtunternehmen

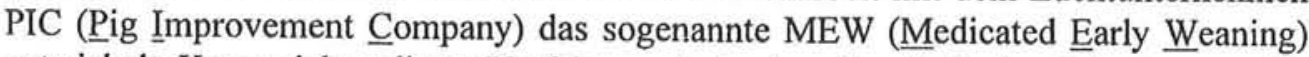
entwickelt. Kennzeichen dieses Verfahrens sind neben dem Isolierten Frühabsetzen die zusätzliche Medikation der Sauen und Ferkel. Diese kann über eine Immunisierung der Sauen gegenüber bestimmten Erregern bzw. auch über eine Immunsuppression und Antibiotika-Behandlungen der Ferkel geschehen. HARRIS (u.a., 1990) hat dann später dieses Verfahren insofern modifiziert, dass er den Haupteffekt in der frühen isolierten Aufzucht der Ferkel erkannte und somit auf die aufwendige Medikation verzichtet wurde. Aus der Bezeichnung MMEW (Modified Medicated Early Weaning) wurde dann die Bezeichnung SEW gewählt und unter der Markenbezeichnung Isowean ${ }^{\circledR}$ durch die Firma PIC propagiert. Das Prinzip des SEW setzt neben dem Frühabsetzen und einer spezifischen Fütterung die separate Ferkelerzeugung, Aufzucht und Mast an möglichst weit voneinander getrennten Standorten voraus, wobei die Schweine in Altersgruppen nach dem Rein-Raus-Prinzip und einem der Bestandsgröße und Säugezeit angepassten Produktionsrhythmus (z.B. Wochenrhythmus) auf die verschiedenen Standorte verteilt werden (Multi-Site-Produktion = Multiple Isolated Sites). Schwerpunktmäßig sollte sich dieser Beitrag mit der tierschützerischen Beurteilung dieses Verfahrens befassen, wobei Aspekte der Tiergesundheit und Produktivität, des Verhaltens von frühabgesetzten Ferkeln und der Sauenfruchtbarkeit näher angesprochen werden.

\section{Tiergesundheit und Produktivität}

Unmittelbar nach der Geburt nehmen Ferkel Antikörper über die Sauenmilch auf und sind dadurch überwiegend passiv gegenüber Krankheitserregern geschützt. Mit zunehmendem Alter (etwa ab dem 10. Lebenstag; siehe Abb.) setzen sich die Ferkel ver- 


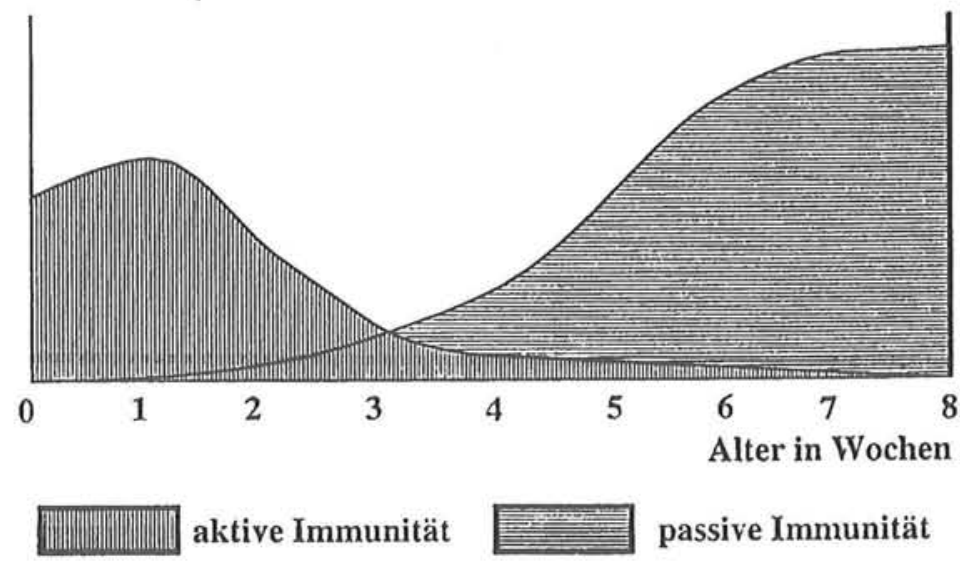

Abb.: Entwicklung der Immunitat beim Ferkel (Development of immunity in the pig)

stärkt aktiv über die körpereigene Immunaktivierung mit Krankheitserregern auseinander, wobei ab etwa der 3. Lebenswoche dieser Anteil der aktiven gegenuber der passiven Immunität überwiegt. In der internationalen Literatur werden in diesem Zusammenhang unterschiedliche Empfehlungen für das optimale Absetzalter von Ferkeln angegeben, um diese durch die isolierte Haltung vor einer vertikalen Übertragung von Krankheitserregern aus dem Zuchtbereich zu schützen. Die Empfehlung müsste unter diesem Gesichtspunkt dahin gehen, so früh wie möglich abzusetzen, da die Ferkel ab einem Alter von etwa 10 Tagen schon für eine ganze Reihe von bedeutsamen Erregern empfänglich sind (Tab. 1). Gegenüber einigen Erregern (sogenannten Frühinfizierern) sind die Ferkel jedoch schon unmittelbar in den ersten Lebenstagen empfänglich (u.a. Streptococcus suis, Actinobacillus suis, B. bronchiseptica), so dass eine vollständige Eliminierung des Krankheitsgeschehens auch beim extremen Frühabsetzen nicht gelingt.

\section{Tabelle 1}

Empfohlenes Absetzalter für die Eliminierung verschiedener Krankheiten (modifiziert nach HARRIS, 1993; PLONAIT \& BICKHARDT, 1997) (Suggested weaning age for the elimination of several diseases (modified after HARRIS, 1993; PLONAIT \& BICKHARDT, 1997))

\begin{tabular}{cc}
\hline Erreger & Alter (Tage) \\
\hline Pasteurella multocida & $<10$ \\
Mycoplasma hypopneumoniae & $<10$ \\
Actinobacillus pleuropneumonia & $<16$ \\
TGE Virus, PRRS & $<21$ \\
Schweinedysenterie, Parvovirose & $<21$ \\
\hline
\end{tabular}

Neben den für die Tiergesundheit relevanten Aspekten ist die aktive Auseinandersetzung mit Erregern aus der Haltungsumwelt mit einem erhöhten Energieaufwand verbunden und beeinflusst das Futteraufnahmeverhalten (JOHNSON und VON BORELL, 1994; DRITZ et al., 1996), so dass Ferkel, ohne dabei notwendigerweise klinisch auffällig zu erscheinen, verminderte Wachstumsraten aufweisen. Anfang der 90'er Jahre belegten Untersuchungen aus der Arbeitgruppe von STAHLY (u.a. WILLIAMS et al., 
Tabelle 2

Futteraufnahme, Wachstum und Futterverwertung von Schweinen unter dem Einfluß einer Immunaktivierung (Alter: 26 - 29 Tage; $\mathrm{n}=2 \times 4 \times 6$; WILLIAMS et al., 1993) (Impact of immune system activation on feed intake, growth rate and feed utilisation of pigs (days 26 to 29 of age; $n=2 \times 4 \times 6$; WILLIAMS et al., 1993)) Lysingehalt \%

\begin{tabular}{|c|c|c|c|c|c|}
\hline Parameter & Aktivierung & 0,60 & 0,90 & 1,20 & 1,50 \\
\hline \multicolumn{6}{|l|}{ Gewicht (kg) } \\
\hline \multirow[t]{2}{*}{ Tag 0} & Niedrig & 6,2 & 6,1 & 6,2 & 6,2 \\
\hline & Hoch & 5,9 & 5,9 & 6,0 & 5,9 \\
\hline \multirow[t]{2}{*}{ Tag $33^{b, c}$} & Niedrig & 20,9 & 26,5 & 27,4 & 28,4 \\
\hline & Hoch & 14,5 & 20,5 & 24,1 & 20,4 \\
\hline \multicolumn{6}{|l|}{ Wachstum und Futterverwertung } \\
\hline \multirow[t]{2}{*}{ Tăgl. Futteraufnahme ${ }^{\mathrm{c}}(\mathrm{kg})$} & Niedrig & 0,99 & 1,11 & 1,10 & 1,10 \\
\hline & Hoch & 0,69 & 0,88 & 0,93 & 0,78 \\
\hline \multirow[t]{2}{*}{ Tägl, Zunahme ${ }^{\text {be }}(\mathrm{kg})$} & Niedrig & 0,44 & 0,62 & 0,64 & 0,67 \\
\hline & Hoch & 0,25 & 0,43 & 0,53 & 0,43 \\
\hline \multirow[t]{2}{*}{ Futterverwertung ${ }^{d} \mathrm{~F} / \mathrm{G}(\mathrm{kg} / \mathrm{kg})$} & Niedrig & 2,23 & 1,81 & 1,72 & 1,63 \\
\hline & Hoch & 2,91 & 2,05 & 1,76 & 1,85 \\
\hline
\end{tabular}

Tabelle 3

Zusammenstellung der Tiergesundheitsbefunde und der Mastleistungen von Schweinen (HÖRÜGEL und SCHIMMEL, 2000) (Compiled list of health parameters and performance of pigs (HÖRÜGEL and SCHIMMEL, 2000))

\begin{tabular}{|c|c|c|c|c|c|c|}
\hline & \multicolumn{4}{|c|}{ Absetzalter } & \multirow{2}{*}{\multicolumn{2}{|c|}{$\begin{array}{c}\text { Einstallung Mast } \\
75 \text { Tage }\end{array}$}} \\
\hline & \multicolumn{2}{|c|}{10 Tage } & \multicolumn{2}{|c|}{20 Tage } & & \\
\hline & Versuch & Kontrolle & Versuch & Kontrolle & Versuch & Kontrolle \\
\hline \multicolumn{7}{|c|}{ Schlachtkörperbefunde } \\
\hline $\mathrm{n}$ & 131 & 60 & 60 & 62 & 59 & 111 \\
\hline Lunge o.b.B. & $93,9 \%$ & $43,3 \%$ & $98,3 \%$ & $29,7 \%$ & $100 \%$ & $58,6 \%$ \\
\hline Pleuritis & - & $6,7 \%$ & - & $14,5 \%$ & $1,7 \%$ & - \\
\hline Pericarditis & $3,1 \%$ & $1,7 \%$ & - & $3,2 \%$ & $3,4 \%$ & $0,9 \%$ \\
\hline \multicolumn{7}{|c|}{ Leistungen } \\
\hline $\mathrm{n}$ & 158 & 60 & 60 & 62 & 59 & 111 \\
\hline Schlachtalter & 177 & 203 & 180 & 206 & 172 & 195 \\
\hline Mastendmasse & $120,3 \mathrm{~kg}$ & $118,8 \mathrm{~kg}$ & $116,3 \mathrm{~kg}$ & $114,1 \mathrm{~kg}$ & $120,5 \mathrm{~kg}$ & $120,0 \mathrm{~kg}$ \\
\hline LTZ & $675 \mathrm{~g}$ & $580 \mathrm{~g}$ & $646 \mathrm{~g}$ & $554 \mathrm{~g}$ & $693 \mathrm{~g}$ & $609 \mathrm{~g}$ \\
\hline MTZ & $851 \mathrm{~g}$ & & $808 \mathrm{~g}$ & $644 \mathrm{~g}$ & $934 \mathrm{~g}$ & $757 \mathrm{~g}$ \\
\hline FA & 2,67 & & 2,78 & & & \\
\hline MFA $\%$ & & & 53,3 & 55,1 & 50,4 & 53,9 \\
\hline
\end{tabular}

1993, Tab. 2), dass eine Immunaktivierung in Abhängigkeit von bestimmten Futterinhaltsstoffen (u.a. vom Lysingehalt) zu einem verminderten Wachstum und einer geringeren Futterverwertung führen. Zudem ließen sich durch eine dem Wachstum angepasste Fütterung mit speziellen Futtermitteln (z.B. auf der Basis von sprühgetrockneten natürlich gewonnenen Plasmaproteinen) eine fruhzeitige Verfettung und ein Auseinan- 
derwachsen der Ferkel reduzieren. Bei entsprechendem Hygienemanagement (MuliSite-Produktion) lassen sich jedoch auch bei mit 3 Wochen abgesetzten Ferkeln annähernd vergleichbar hohe Wachstumsleistungen erzielen (HÖRÜGEL und SCHIMMEL, 2000, Tab. 3). Diese Erfahrungen lassen vermuten, dass eine Unterbrechung der horizontalen Erregerkette von entscheidender Bedeutung für die Produktivität zu sein scheint, sofern die Ferkel nicht zu lange Säugezeiten ( $>3$ Wochen) erfahren (Tab. 4).

Tabelle 4

Tiergesundheit und Produktivităt mit SEW (Animal health and productivity with SEW)

\begin{tabular}{ccc}
\hline & Immunstatus & Produktivität \\
\hline Isoliertes Absetzen nach & $\begin{array}{c}\text { Geringe Immunaktivierung durch } \\
\text { vertikale und horizontale Unterbre- } \\
\text { chung der Erregerkette } \\
\text { Immunaktivierung bei horizontaler } \\
\text { Unterbrechung der Erregerkette }\end{array}$ & +++ \\
\hline
\end{tabular}

\section{Verhalten frühabgesetzter Ferkel}

Zum Verhalten frühabgesetzter Ferkel existieren aus der Vergangenheit zahlreiche Versuchsergebnisse (u.a. MARX, 1973; WORSAAE und SCHMIDT, 1980; VON BORELL, 1984) die darauf hindeuten, dass eine frühzeitige Unterbrechung der Kontakte zwischen Ferkeln und Sauen in Verbindung mit der abrupten Futterumstellung zu Verhaltensproblemen wie gegenseitiges Besaugen und belastungsanzeigenden physiologischen Reaktionen führen können. Diese Ergebnisse sind nur bedingt auf die heutigen Verhältnisse beim isolierten Frühabsetzen übertragbar, da bei diesem Verfahren ein anderes Fütterungsmanagement mit einem komplexen, den Bedürfnissen der Ferkel angepassten Futtermittel zum Einsatz kommt. Daher sind auch aktuelle Untersuchungen zu der Problematik des Frühabsetzens nur bedingt verwertbar, wenn die Verhaltensdaten aus vergleichenden Verhaltensuntersuchungen bei unterschiedlichem Absetzalter ohne räumliche Isolierung und unter Einsatz eines konventionellen Futters erhoben wurden (WOROBEC et al. 1999, HOHENSHELL et al., 2000). Es zeigte sich jedoch auch in Untersuchungen unter den Bedingungen des SEW, dass beim Frühabsetzen ( $<3$ Wochen) das gegenseitige Bewühlen bzw. Kauen im Bauchbereich und an anderen Körperteilen vermehrt zu beobachten ist (GONYOU et al., 1998; ROBERT et al., 1999, YUAN et al., 1999), und dass nach dem Absetzen im Alter zwischen 9 und 13 Tagen die Cortisolkonzentrationen gegenüber gleichaltrigen nicht abgesetzten Ferkeln um das 3-4-fache erhöht waren (YUAN et al., 1999). Dabei scheint sich eine komplexe Futterration (im Vergleich zu herkömmlichem Futter) erst ab einem Absetzalter von 4 Wochen reduzierend auf die Belastungsreaktionen der Ferkel auszuwirken (WEARY et al., 1999). Die Proteinquelle im Futter scheint wiederum das Futteraufnahmeverhalten nach dem Absetzen insofern zu beeinflussen, dass sich die Futteraufnahme durch komplexe Rationen mit getrockneten Plasmaproteinen gegenüber Magermilchpulver als Proteinquelle erhöhen lässt (ERMER et al., 1994). Die Untersu- 
chungen von GONYOU et al. (1998) ließen Langzeiteffekte des Absetzalters auf das gegenseitige Bewühlen und das Kauen von Körperteilen erkennen. Ferkel, die nach 12 Tagen abgesetzt wurden, wiesen auch als Mastschweine in der Endmastperiode gegenüber Schweinen, die nach 21 Tagen abgesetzt wurden, erhöhte Zeitanteile für diese Verhaltensmerkmale auf (Tab. 5).

Tabelle 5

Einfluß des Absetzalters auf Zeitanteile des Verhaltens (\%) wăhrend der Mastperiode von Schweinen (modifiziert nach GONYOU et al., 1998) (Influence of weaning age on behavioural time budgets (\%) during the fattening period of pigs (modified after GONYOU et al., 1998))

\begin{tabular}{|c|c|c|c|}
\hline Merkmal & 12 Tage & $\begin{array}{l}\text { Absetzalter } \\
21 \text { Tage }\end{array}$ & $\mathrm{P}$ \\
\hline Fressen & 7,81 & 8,27 & ns \\
\hline Trinken & 2,43 & 2,07 & ns \\
\hline Stehen (inaktiv) & 23,64 & 25,76 & ns \\
\hline \multicolumn{4}{|l|}{ Bewulhlen anderer } \\
\hline Schweine & 0,755 & 0,476 & 0,05 \\
\hline Kauen anderer Schweine & 1,146 & 0,843 & 0,05 \\
\hline Kauen von Objekten & 0,000 & 0,021 & 0,05 \\
\hline
\end{tabular}

Sauenfruchtbarkeit bei verkürzter Laktationsdauer

Zur Reproduktionsleistung von Sauen bei verkürzter Laktationsdauer liegen zahlreiche ältere und neuere Untersuchungen vor (u.a. MARX und HOEPFNER, 1976; XUE et al., 1993; BILKEI und BIRO, 1998). Auf der Basis eines sehr umfangreichen Datenmaterials berichteten XUE et al. (1993), dass sich mit zunehmender Laktationsdauer (bis zu 4 Wochen) die Wurfgröße erhöht, das Absetz-Oestrus-Intervall verkürzt und sich das Intervall zwischen aufeinander folgenden Geburten verlängert, so dass die Gesamtzahl lebend geborener Ferkel pro Sau und Jahr prinzipiell nicht von der Laktationsdauer beeinflusst wird. Dies hängt mit dem kompensatorischen Effekt einer verkürzten Laktation (z.B. 17 Tage) auf diesen Produktionsparameter zusammen. Bei einer Laktationsdauer von weniger als 3 Wochen wirken sich jedoch die notwendigen Involutions- und Restitutionsvorgänge am Endometrium post partum limitierend auf die Reproduktionsleistung aus. Im Zusammenhang mit dem SEW wird dennoch von einer Verbesserung der Sauenproduktivität gesprochen (DIAL et al., 1992), wobei die Anzahl abgesetzter Ferkel pro Sau und Jahr sowie die Anzahl Geburten pro Sau und Jahr bei Sauen mit einer Säugedauer von unter 3 Wochen nicht notwendigerweise gegenüber den Leistungen von herkömmlich abgesetzten Sauen erhöht ist. Maßgeblich für die hohe Produktivität scheinen eher die mit dem Verfahren verbundenen Haltungs, Hygiene- und Fütterungsmanagementmaßnahmen zu sein. Beim extremen Frühabsetzen (z.B. < 2 Wochen) können die Fruchtbarkeitsprobleme zu höheren Merzungsraten bei den Sauen führen (XUE et al., 1993). BILKEI und BIRO (1998) konnten bei einem Vergleich der Merzungsraten zwischen Sauen mit herkömmlicher Säugezeit (21-28 Ta- 
gen und nach dem SEW-Verfahren abgesetzter Sauen (14-17 Tagen) keine signifikanten Unterschiede bezüglich zehn ausgewerteter Merzungskriterien feststellen. Insgesamt liegen jedoch zu der speziellen Problematik der Fruchtbarkeit und Nutzungsdauer von Sauen im SEW-Verfahren nur wenige Untersuchungen vor.

\section{Tierschützerische Beurteilung des Absetzalters und Schlussfolgerungen}

Die deutschen (SCHWEINEHALTUNGSVERORDNUNG, 1994) und europäischen (EU-RICHTLINIE 91/630) Tierschutzvorschriften verbieten das Absetzen von unter 21 Tage alten Ferkeln. Diese Vorschriften beziehen sich auf Empfehlungen aus wissenschaftlichen Untersuchungen auf der Basis herkömmlicher Aufzuchtverfahren. Im Bericht der EU- Expertengruppe zur Einschätzung des Wohlbefindens intensiv gehaltener Schweine für das SVC (SCIENTIFIC VETERINARY COMMITTEE; 1997) wird die Problematik des Frühabsetzens erneut vor dem Hintergrund des SEW-Verfahrens behandelt. Danach sollte SEW nur erlaubt werden, wenn nachgewiesen werden kann, dass die Vorteile des SEW bezüglich des Wohlbefindens von Sauen und Ferkeln gegenüber den Nachteilen überwiegen (Kriterien: Mortalität, Durchfallerkrankungen, Wachstumsrate, Indikatoren des Verhaltens wie z.B. das gegenseitige Besaugen). Generell lassen die Empfehlungen zum Absetzalter viel Spielraum: Vor dem 28. Tag sollte nicht abgesetzt werden. Aus praktischen Gründen verbunden mit Gesundheitsmaßnahmen können Ferkel auch bis zu 7 Tage früher abgesetzt werden. Einzelne Ferkel können aus medizinischen Gründen (z.B. kranke Sau, Agalaktie) auch früher $(<21$ Tage) abgesetzt werden.

Unter Einbeziehung der neueren Literatur zur Beurteilung des SEW werden folgende Schlussfolgerungen gezogen:

- Die Produktivitäts- und Gesundheitsvorteile beim extremen Frühabsetzen $(<21$ Tage) sind gegenüber Verfahren unter Beachtung des gesetzlich zulässigen Mindestabsetzalters der Ferkel bei konsequenter Umsetzung von Gesundheitsmanagementmaßnahmen (u.a. Multi-Site-Produktion nach dem Rein-Raus-Prinzip) als eher gering einzustufen.

- In neueren Untersuchungen zum Verhalten von frühabgesetzten Ferkeln wird unter Tierschutzgesichtspunkten von einer Unterschreitung des Absetzalters von 3 bis 4 Wochen abgeraten.

- Eine abschließende Bewertung hängt u.a. von der Gewichtung und Interpretation der für das Wohlergehen von Sauen und Ferkeln relevanten Indikatoren (Gesundheit, Leistung, Verhalten etc.) ab.

- Solange Zweifel an der Unbedenklichkeit einer sehr frühen Isolation der Ferkel von der Sau bestehen, und sich durch das extreme Frühabsetzen Fruchtbarkeitsstörungen bei den Sauen einstellen, sollte gemäß der EU-Empfehlungen an einem Mindestabsetzalter von 3 bis 4 Wochen festgehalten werden. 


\section{Literatur}

ALEXANDER, T.J.L.; THORNTON, K; BOON, G.; LYSONS, R.J.; GUSH, A.F.:

Medicated early weaning to obtain pigs free from pathogens endemic in the herd of origin. Vet. Rec. 106 (1980), 114-119

BILKEI, G.; BIRO, O:

Erfahrungen mit dem getrennten Fruhabsetzen (Segregated Early Weaning, SEW) der Schweine. Berl. Munch. Tierărztl. Wschr. 111 (1998), 326-331

VON BORELL, E.:

Vergleichende Kortisolbestimmungen im Blut konventionell gehaltener und fruhabgesetzter Ferkel in Flatdecks mit verschiedenen Flächengroßen und Bodenarten. Univ. Hohenheim, Diplomarbeit, 1984

DIAL, G.D.; MARSH, W.E.; POLSON, D.D.; VAILLANCOURT, J.P.:

Reproductive failure : Differential diagnosis. In : LEMAN, A.D.; STRAW, B.E.; MENGELING, W.L.; D'ALLAIRE, S.D.; TAYLOR, D.J.. Diseases of Swine, $7^{\text {th }}$ ed., Wolfe Publ. London (1992), 88-138

DRITZ, S.S., OWEN, K.Q.; GOODBAND, R.D.; NELSSEN,, J.L.; TOKACH, M.D.; CHENGAPPA, M.M.; BLECHA, F:

Influence of lipopolysaccharide-induced immune challenge and diet complexity on growth performance and acute-phase protein production in segregated early-weaned pigs. J. Anim. Sci. 74 (1996), 1620-1628

ERMER, P.M., MILLER, P.S.; LEWIS, A.J.:

Diet preference and meal patterns of weanling pigs offered diets containing either spray-dried porcine plasma or dried skim milk. J. Anim. Sci. 72 (1994), 1548-1554

GONYOU, H.W.; BELTRANENA, E.; WHITTINGTON, D.L.; PATIENCE, J.F.:

The behaviour of pigs weaned at 12 and 21 days of age from weaning to market. Can. J. Anim. Sci. 78 (1998), 517-523

HARRIS, D.L.:

The use of Isowean® 3-site production to upgrade health status. Proc. $11^{\text {th }}$ Intl. Congr. Pig Vet. Soc. (1990), 374

HOHENSHELL, L.M.; CUNNICK, J.E.; FORD, S.P.; KATTESH, H.G.; ZIMMERMAN, D.R.; WILSON, M.E.; MATTERI, R.L.; CARROLL, J.A.; LAY,D.C.:

Few differences found between early- and late-weaned pigs raised in the same environment. J. Anim. Sci. 78 (2000), 38-49

HÖRÜGEL, K.; SCHIMMEL, D.:

Multisite-Produktion - ein Verfahren zur Verbesserung der Tiergesundheit. Prakt. Tierarzt 81 (1), (2000), 61-70

JOHNSON, R.W.; VON BORELL, E.:

Lipopolysaccharide-induced sickness behavior in pigs is inhibited by pretreatment with indomethacin. J. Anim. Sci. 72 (1994), 309-314

MARX, D.:

Vergleichende Untersuchungen über das Verhalten von Saugferkeln und fruhabgesetzten Ferkeln in Kăfiggruppenhaltung (Batteriehaltung). Berl. Munch. Tierärztl. Wschr. 86 (1973), 289-296, 301-306

MARX, D.; HOEPFNER, G.:

Reproduktionsleistung von Sauen nach unterschiedlicher Dauer der Laktationszeit und bei hormoneller Brunstauslðsung nach 14-bis 19-tăgiger Laktation. Zuchtungskunde 48 (1976), 29-44

PLONAIT, H.; BICKHARDT, K.: Lehrbuch der Schweinekrankheiten. 2., neubearb. Auflage, Parey Buchverlag Berlin (1997), p 556

RICHTLINIE DES RATES ƯBER MINDESTANFORDERUNGEN FUR DEN SCHUTZ VON SCHWEINEN: (1991; 91/630/EWG). Amtsblatt der Europäischen Gemeinschaften, Nr. L 340/33

ROBERT, S.; WEARY, D.M.; GONYOU, H.W.:

Segregated early weaning and welfare of piglets. J. Appl. Anim. Welf. Sci. 2 (1999), 31-40

SCIENTIFIC VETERINARY COMMITTEE:

Report on The Welfare of Intensively Kept Pigs by VON BORELL, E.; BROOM, D.M.; CSERMELY, D.; DIJKHUIZEN, A.A.; EDWARDS, S.A.; JENSEN, P.; MADEC, F.; STAMATARIS, C.; Doc XXIV/B3/ScVC/0005/1997, Brussels, Internet Publication (1997), page 1-191

(http://europa.eu.int/comm/dg24/health/sc/oldcomm4/previous en.html)

VERORDNUNG ZUM SCHUTZ VON SCHWEINEN BEI STALLHALTUNG:

(Schweinehaltungsverordnung, 1994). Bundesgesetzblatt, Jahrgang 1988, 673-675, geăndert 1994, Bundesgesetzblatt I, 312 
Arch. Tierz. 43 (2000) 4

WEARY, D.M.; APPLEBY, M.C.; FRASER, D.:

Responses of piglets to early separation from the sow. Appl. Anim. Behav. Sci. 64 (1999), 289-300

WILLIAMS, N.H.; STAHLY, T.S.; ZIMMERMAN, D.R.; WANNEMUEHLER, M.:

Impact of immune system activation and amino acid regimen on growth rate, efficiency of feed utilization and body nutrient accretion in pigs. Swine Research Report, Iowa State University (1993), 12-14

WOROBEC, E.K.; DUNCAN, I.J.H.; WIDOWSKI, T.M.:

The effects of weaning at 7, 14 and 28 days on piglet behaviour. Appl. Anim. Behav. Sci. 62 (1999), 173-182

WORSAAE, H.; SCHMIDT, M.:

Plasma cortisol and behaviour in early weaned piglets. Acta vet. scand. 21 (1980), 640-657

XUE, J.L.; DIAL, G.D.; MARSH, W.E.; DAVIES, P.R.; MOMONT, H.W.:

Influence of lactation length on sow productivity. Livest. Prod. Sci. 34 (1993), 253-265

YUAN, Y; TAUCHI, M.; ZANELLA, A.J.:

The behavioral and physiological responses of pigs to segregated early weaning. J. Anim. Sci. 77 (1999), Suppl. 1, 35

Eingegangen: 25.05 .2000

Akzepticrt: 26.06 .2000

Anschritt des Verfassers

Prof. Dr. EBERHARD VON BORELL

Institut fur Tierzucht und Tierhaltung mit Tierklinik der

Martin-Luther-Universităt Halle-Wittenberg

Adam-Kuckhoff-Str. 35

D-06108 Halle/S.

E-Mail: BORELL@landw.uni-halle.de 
Arch. Tierz., Dummerstorf 43 (2000) 4, 346

\title{
Buchbesprechung
}

\section{Krankheiten der Reitpferde}

\author{
PETER LAUNER, JƯRGEN MILL und WILFRIED RICHTER
}

2., völlig neu bearbeitete und erweiterte Auflage, 384 Seiten, 211 Farbfotos, 119 Zeichnungen, 28 Tabellen, Verlag Eugen Ulmer, Stuttgart, 1999, ISBN 3-8001-7394-8, 78,00 DM

In einer Zeit zunehmender Technisierung und elektronischer Medien in allen Arbeits- und Lebensbereichen, erhält die Beziehung vieler Menschen zum Pferd einen immer größeren Stellenwert. Neben der berufsmäßigen Reiterei wächst die Zahl der Freizeitreiter ständig und damit auch das Bedurfnis mehr uber den richtigen Umgang mit dem Pferd, auch dem erkrankten, zu wissen. Diesem Trend folgend und wissend, dass nur ein gesundes Pferd ein Optimum an Leistungsfahigkeit erbringt, haben die Autoren dieses Buch in nunmehr zweiter Auflage neu bearbeitet vorgelegt. Es ist in erster Linie fur Reiter, Trainer, Halter, Zuchter und Freunde von Sportpferden bestimmt und soll einerseits einen Überblick der wichtigsten Erkrankungen von Sportpferden vermitteln und andererseits dem Pferdehalter ermöglichen auch vorbeugend wirksam werden zu können, um die Erkrankungshäufigkeiten der Pferde zu senken. Es kann und soll nicht den Tierarzt ersetzen, hilft jedoch Pferdehaltern und -nutzern Krankheitssymptome fruhzeitig zu erkennen, Krankheiten vorzubeugen und ermøglicht eine effektive Zusammenarbeit mit dem Tierarzt.

Wie wichtig Haltung, Futterung und der sachkundige Umgang mit dem Pferd sind, wird in den ersten Buchabschnitten dargelegt, erganzt durch den Abschnitt der Beschreibung der Untersuchungsmethoden. Neu in diesem Buchteil ist z. B. das Kapitel „Tierschutz im Pferdesport" und gegenuber der ersten Auflage wesentlich erweitert wurde das Kapitel „Doping und Dopingkontrolle". Der aus drei weiteren großen Abschnitten bestehende Hauptteil des Buches widmet sich der Beschreibung der hăufigsten Krankheiten des Pferdes. So findet der Leser die Beschreibungen der Infektionskrankheiten getrennt nach Viruskrankheiten und bakteriellen Infektionen. Es folgen die Stoffwechselstörungen, Mangelkrankheiten und Vergiftungen und der große Abschnitt der Erkrankung bestimmter Organsysteme von den Krankheiten der Haut, der Atmungsorgane bis zum Nervensystem und den Verhaltensstörungen. Jede dieser Krankheiten wird ausfuhrlich beschrieben, ihre Ursache, das Erscheinungsbild, Behandlung und Vorsorge dargestellt. Die letzten Abschnitte des Buches sind der Versorgung des erkrankten Pferdes, einer umfangreichen Übersicht zu den Krankheitsanzeichen und einem tabellarischen Anhang zu Kenn- und Normwerten gewidmet.

Das Buch ist kenntnisreich aufgebaut und zeigt, dass die Autoren den Stoff souverăn beherrschen. Es hält für Pferdezuchter, -freunde und -liebhaber aber auch Tierärzte eine Fulle von sorgfáltig zusammengetragenen Informationen mit hoher Praxisrelevanz bereit und verschaff einen umfangreichen Überblick uber die bemerkenswerte Vielfalt von Erkrankungen des Reitpferdes. Drucktechnische Hervorhebungen, zahlreiche Zeichnungen, vor allem die Fulle ausgezeichneter Farbfotos, unterstutzen die informativen und klaren Textaussagen. Sowohl die große Sachkompetenz der Autoren als auch die hervorragende Ausstattung durch den Verlag machen dieses Buch zu einem unverzichtbaren Handbuch und Nachschlagewerk fur den ambitionierten Pferdefreund. Es ist uneingeschränt vielen Lesern zu empfehlen. 